\title{
Editorial: ASD and Illegal Behaviors
}

\author{
Marc Woodbury-Smith
}

Published online: 13 September 2014

(C) Springer Science+Business Media New York 2014

\begin{abstract}
The last decade has seen significant advances in our understanding of autism spectrum disorder, from both biological and clinical perspectives. Outcome runs the gamut from complete independence to relative dependence. Of particular interest, among those described with poor outcome are a small number of individuals, male and female, who have engaged in unlawful behavior. This special issue of the Journal of Autism and Developmental Disorders presents a number of papers providing further insight into this issue.
\end{abstract}

Keywords Autism spectrum disorder (ASD) .

Adulthood $\cdot$ Outcome $\cdot$ Criminal behaviour

The last decade has seen significant advances in our understanding of autism spectrum disorder (ASD), from both biological and clinical perspectives, and the Journal of Autism and Developmental Disorders has been at the forefront of publishing research that has facilitated a deeper and more integrated knowledgebase concerning ASD's etiology, diagnosis, treatment and outcome. Moreover, whilst historically much of the research was focused on the lives of children, there has now been a shift to consider also the lives of adults with this diagnosis. A key to understanding the life history of ASD is the longitudinal study design, and it was Sula Wolf's description of 'loners' that first sparked my interest in adult outcome among those with childhood socio-communicative impairments (Wolff

M. Woodbury-Smith $(\square)$

Department of Psychiatry and Behavioural Neurosciences, McMaster Children's Hospital, McMaster University, Chedoke Campus (Evel Building, Room 457), Hamilton, ON, Canada

e-mail: woodbur@mcmaster.ca
1995). This highly engaging text describes the life course of a cohort of children designated as 'loners' during childhood (or in Wolf's terminology, 'schizoid personality in childhood'), and follows them into the middle adult years. What is striking is that outcome runs the gamut from complete independence to relative dependence. Of particular interest, among those described with poor outcome were a small number of individuals, male and female, who had engaged in unlawful behavior. At about the same time, clinical cohorts were described by Wing (1981) and Tantam (1988a, b) among whom a small number of individuals had engaged, or were alleged to have engaged, in illegal behaviors.

Since these early descriptions a number of case studies have been published describing individuals with ASD who have engaged in a variety of illegal behaviors, and which span the full range of severity. What makes these descriptions particularly important is that a causal relationship is often hypothesized between the core clinical and neuropsychological characteristics of the ASD and the offences committed. Case studies are, of course, limited in their ability to more formally test these hypotheses, and thereby offer evidence concerning factors associated with illegal behaviors in the ASD population more generally. Indeed, as I and a colleague discuss in this current edition, despite the not insignificant number of publications focused on offending and illegal behaviors among those with ASD, there remains a dearth of available information from which clinicians can plan treatment, and services can be commissioned. This current edition of the Journal of Autism and Developmental Disorders presents a number of papers concerning unlawful behavior among adolescents and adults with ASD, and several other articles on this topic have appeared in this journal over the last year. These each bring their own unique perspective on this topic. 
Cheely et al. (2012) directly examined the prevalence of youth with ASD in the criminal justice system (CJS) using surveillance data and official criminal justice records. Strikingly, $5 \%$ of a geographic population-based sample of youth aged 12-18 years with ASD had a history of contact with the CJS as recorded in the official statistics. Moreover, in comparison to matched controls of juvenile justice-involved youth, the cases were more likely to have offences against another person. This study also identified that individuals with ASD were more likely to be diverted from custodial care into specialist provision. The strength of this study is in its use of epidemiological data, and whilst the figure of $5 \%$ is not particularly high, it does stress the importance of not overlooking the risk of illegal behavior in this population. Additionally, by their sampling approach, cases that may have been diverted from incarceration (as many are) are represented in the prevalence figure. Interestingly, King and Murphy (this edition), in their detailed systematic review of the literature, give consideration to the larger literature concerned with the representation of ASD in the CJS, and arrive at similar conclusions concerning the prevalence, suggesting that "people with ASD are somewhat over-represented in the CJS" although also arguing for the need for more robust research.

Having considered population level data to measure prevalence of unlawful behavior, it is also pertinent to consider the risk in a case control design. Lundström and colleagues (this edition) use a number of formal registers detailing the mental health diagnoses given to individuals within the geographic region of greater Stockholm, to examine the risk of violent criminal behavior in different diagnostic groups and a control group. Strikingly, no association was seen between ASD and violent offending based on a case-control comparison. How, then, can we reconcile the conflicting evidence that emerges from different studies? With current evidence this is a difficult question to answer, and perhaps belies the true importance of this subject, because methodological confounds aside (which are discussed in the respective papers), and even if the true prevalence is only somewhat higher than in the general population, a more important question in my mind is the extent to which the risk of criminal behavior in this population is driven by factors related to the core features of ASD.

A small number of papers have previously considered this (discussed in Woodbury-Smith and Dein in this volume), and these are complemented by the study of Buon et al. (2013). In their study intentional judgment is examined among individuals with ASD in a case-control design. Interestingly, the ASD subjects were able to correctly judge the distress of victims in the research scenarios, suggesting an intact empathy mechanism. However, the ASD subjects displayed difficulty judging intention (i.e. whether an action is accidental or purposeful), and in making moral judgments. One important observation made is that different patterns of cognitive strength and vulnerability were characteristic of different sub-groups, in support of the notion that illegal behaviours may be characteristic of a subgroup within the autism spectrum that is distinguished by its own unique pattern of cognitive impairments.

One significant limitation of the research so far has been the small volume of clinical and treatment based research. However, Maras and Bowler (this edition) have undertaken a detailed review of testamentary capacity in ASD, that, importantly, fouses on capacity in the CJS for a person with ASD who is a victim of crime. The risk of being a victim of crime has certainly been raised in the ASD literature, and cases are described in the media, but little consideration has been given to some of the issues an individual with ASD may face when giving their eyewitness account of events. Maras and Bowler do conclude that this population is no more suggestible than the population at large, and are quite capable of accurate recall. Nonetheless, despite these cognitive strengths, it is argued that a person with ASD may be deemed to lack credibility due to the way they may present themselves (for example, their communication style, appearance, posture and body movement). As such, special consideration may need to be given during both the police interview and the criminal proceedings to ensure that their testimony is given the weight it deserves. Interestingly, this very issue is being discussed with increased frequency on internet forums, and it is hoped that there may now be enough momentum and interest to ensure that recommendations and guidelines become available.

Treatment implications have also been considered by Post and colleagues (this edition). In their review, the authors focussed on the risk of stalking among individuals with ASD, an important risk behavior to consider in this population as discussed in a previous JADD article (Stokes et al. 2007). Although stalking per se may not be particularly common, inappropriate attempts to form relationships may be much more commonly seen. Their article expounds the assessment process and treatment options, with consideration given to individual and group based treatment modalities, and with a particular focus on video modeling.

Without doubt, these articles, and similar ones published elsewhere, are facilitating a better understanding of unlawful behavior among individuals with ASD into 'evidence' that has direct implication for the management of such individuals in clinical and criminal justice settings. There is still much to be learned, and inconsistencies in 
results between different studies need to be adequately explained. Most notably, prevalence estimates remain wide for both unlawful behavior in this population as well as numbers of individuals with ASD incarcerated in forensic and other criminal justice settings. There is also a great need for ASD expertise to be shared with criminal justice workers. Finally, there needs to be clear service pathways and management guidelines for those individuals with ASD who have engaged in unlawful behavior such that their rehabilitation needs are addressed and to avoid further compounding their social exclusion by incarceration, essentially without treatment, in either long stay forensic institutions or jails. A research colleague and I have written a review article in this edition of the journal discussing these various issues, and proposing a new research agenda that more directly informs service provision and development.

Acknowledgments M.W.S. acknowledges the support of the Canadian Institute for Health Research (CIHR) and Scottish Rite Charitable Foundation during the preparation of this manuscript.

\section{References}

Buon, M., Dupoux, E., Jacob, P., Chaste, P., Leboyer, M., \& Zalla, T. (2013). The role of causal and intentional judgments in moral reasoning in individuals with high functioning autism. Journal of Autism and Developmental Disorders, 43, 458-470.

Cheely, C. A., Carpenter, L. A., Letourneau, E. J., Nicholas, J. S., Charles, J., \& King, L. B. (2012). The prevalence of youth with autism spectrum disorders in the criminal justice system. Journal of Autism and Developmental Disorders, 42(9), 1856-1862.

Stokes, M., Newton, N., \& Kaur, A. (2007). Stalking, and social and romantic functioning among adolescents and adults with autism spectrum disorder. Journal of Autism and Developmental Disorders, 37, 1969-1986.

Tantam, D. (1988a). Lifelong eccentricity and social isolation. I. Psychiatric, social, and forensic aspects. British Journal of Psychiatry, 153, 777-782.

Tantam, D. (1988b). Lifelong eccentricity and social isolation. II: Asperger's syndrome or schizoid personality disorder? British Journal of Psychiatry, 153, 783-791.

Wing, L. (1981). Asperger's syndrome: a clinical account. Psychological Medicine, 11, 115-129.

Wolff, S. (1995). Loners: The life path of unusual children. London: Routledge. 